\title{
Extracellular Matrix-like Cell-Adhesive Hydrogels from RGD- Containing Poly(ethylene glycol) Diacrylate
}

Junmin Zhu, ${ }^{\dagger}$ Jeffrey A. Beamish, ${ }^{\dagger}$ Chad Tang, ${ }^{\dagger}$ Kandice Kottke-Marchant ${ }^{\ddagger}$ and Roger E. Marchant ${ }^{\dagger, \S, *}$

\author{
${ }^{\dagger}$ Department of Biomedical Engineering, Case Western Reserve University, Cleveland, Ohio 44106 \\ ${ }^{\S}$ Department of Macromolecular Science, Case Western Reserve University, Cleveland, Ohio 44106 \\ ${ }^{\ddagger}$ Department of Clinical Pathology, Cleveland Clinic Foundation, 9500 Euclid Avenue, Cleveland, Ohio 44195
}

\section{Experimental Section}

Materials and Methods. Acryloyl-PEG-NHS (Acr-PEG-NHS, Mw 34000) was purchased from Nektar (Huntsville, AL). Ethylenediamine was purchased from Aldrich and was used as received. All Fmoc-protected amino acids were purchased from AnaSpec Inc. Irgacure 2959 (I2959, 2-hydroxy-1[4-(hydroxyethoxy)phenyl]-2-methyl-1-propane (Ciba Specialty Chemicals, Tarrytown, NY) was used as the water-soluble photoinitiator. MALDI-MS mass spectrometry was performed on a BrukerBiflex III MALDI-TOF mass spectrometer by dissolving the sample and the matrix of 2,5-dihydroxybenzoic acid in 1:1 (v/v) ethanol and water, and mass spectra were typically accumulated from 200 laser shots.

Peptide synthesis. Dap-GRGDSP peptide with an N-terminus amine group was prepared by solidphase peptide synthesis using standard 9-fluoromethoxycarbonyl (Fmoc) chemistry. The peptide was 
generated on a PAL resin (loading $0.38 \mathrm{mmol} / \mathrm{g}$, Applied Biosystems) using an Applied Biosystems Model 433A automatic peptide synthesizer, with O-tert-butyl side chain protection for Asp and Ser, and 2,2,4,6,7-pentamethyldihydrobenzofurn-5-sulfonyl (Pbf) for Arg. In general, couplings were performed with a 4-fold excess of Fmoc-amino acid in the presence of O-(benzotriazol-1-yl)N,N,N'N'-tetramethyluronium hexafluorophosphate (HBTU)/N,N-diisopropylethylamine (DIEA)/1hydroxybenzotrilazole (HOBt) (1:1:0.5, v/v/v) for $30 \mathrm{~min}$. Fmoc groups were cleaved with $20 \%$ piperidine in dimethylformide (DMF) for $10 \mathrm{~min}$. A small amount (100 $\mathrm{mg})$ of peptide-bounded resin was used to cleave the peptide from the resin. Resin cleavage and deprotection were performed with trifluoroacetic acid (TFA)/ethanedithiol/triisopropylsilane/water (91:3:3:3, v/v/v/v) for 2 h. Cleaved resin was removed by filtration, and washed twice with $0.2 \mathrm{ml}$ of TFA. After precipitation of the crude peptide by addition of ice-cold diethyl ether, the peptide was further purified by reverse-phase HPLC. The peptide structure was confirmed by MALDI-TOF MS analysis (as shown in Figure 1a in the Manuscript) with a peak at $\mathrm{m} / \mathrm{z}=673.65$ for $[\mathrm{M}+\mathrm{H}]^{+}$(calculated mass 673.7 ) and a peak at $\mathrm{m} / \mathrm{z}=$ 695.93 for $[\mathrm{M}+\mathrm{Na}]^{+}$(calculated mass 695.7).

Synthesis of PEGDA6K. PEGDA6K was prepared by combining $0.5 \mathrm{mmol} / \mathrm{ml}$ dry PEG6K (Aldrich) (Mw 6,000), $2.0 \mathrm{mmol} / \mathrm{ml}$ acryloyl chloride, and $1.0 \mathrm{mmol} / \mathrm{ml}$ triethylamine in anhydrous dichloromethane and stirring under nitrogen overnight. The resulting product was then precipitated with ether, filtered, and dried in a vacuum oven at room temperature. In comparison with Mw of PEG6K from the MALDI-TOF MS spectra (Figure S1a), Mw of PEGDA6K (Figure S1b) increased by 105, which was corresponding to the attachment of two acryloyl $\left(\mathrm{CH}_{2}=\mathrm{CHCOO}\right)$ group. 

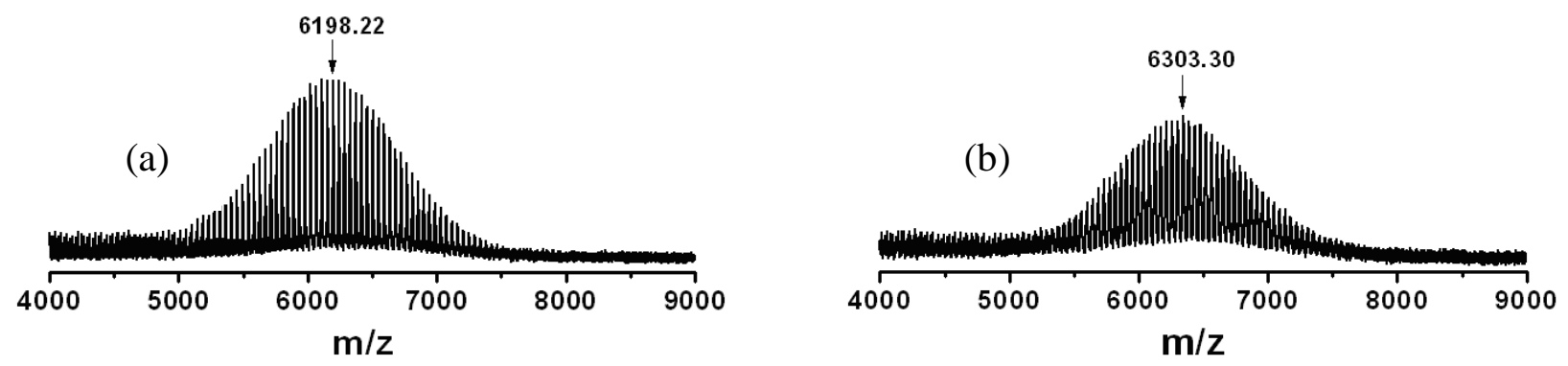

Figure S1. MALDI-TOS MS spectra of (a) PEG6K and (b) PEGDA6K

Synthesis of EDA-PEGDA. Scheme 1 shows the strategy to synthesize EDA-PEGDA. EDA was dissolved in methylene chloride (DCM). Acr-PEG-NHS with two-fold molar amount as EDA, was dissolved in DCM, and added dropwise to the EDA solution. The mixture was stirred at RT for 24h, and then the solvent was removed by rotary evaporation. The raw product was dialyzed against water with membranes of Mw Cutoff 5,000 for 48h. The final dialysis product was lyophilized and further purified using reverse phase HPLC. From the MALDI-TOF MS spectra, as shown in Figure S2, the Mw of EDA-PEGDA (Figure S2b) almost doubled as the starting material of Acr-PEG-NHS (Figure S2a), which confirmed the conjugation of two molecules of Acr-PEG-NHS with one molecule of EDA.

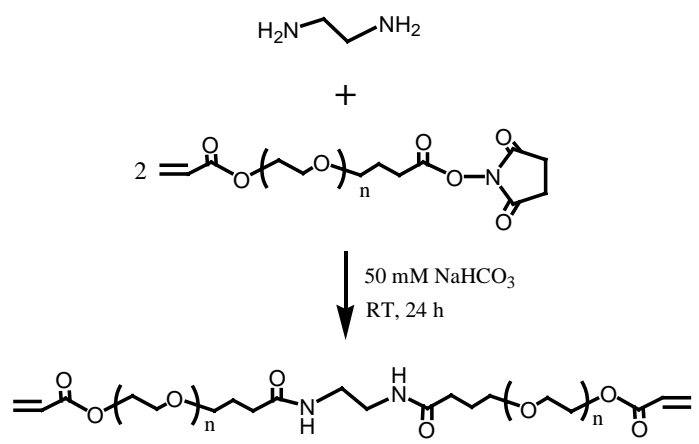

Scheme S1. Synthesis of EDA-PEGDA 

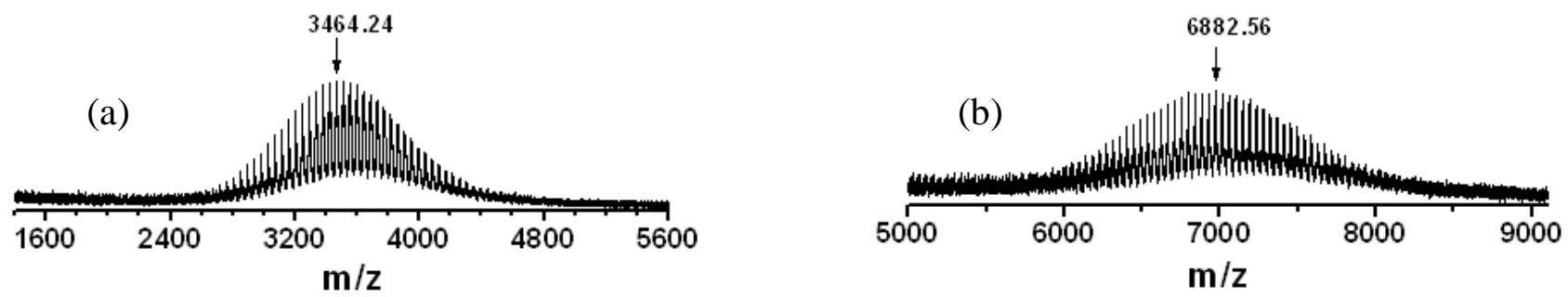

Figure S2. MALDI-TOS MS spectra of (a) Acr-PEG-NHS (Mw 3400) and (b) EDA-PEGDA

Synthesis of RGD-PEGDA. Dap-GRGDSP peptide was dissolved in $50 \mathrm{mM}$ of sodium bicarbonate buffer ( $\mathrm{pH}$ 8.2). Acr-PEG-NHS with two-fold molar amount as the peptide, was dissolved in $50 \mathrm{mM}$ of sodium bicarbonate buffer ( $\mathrm{pH} 8.2$ ), and added dropwise to the aqueous peptide solution. The mixture was stirred at RT for $24 \mathrm{~h}$, and then dialyzed against water with membranes of Mw Cutoff 5,000 for 48h. The MALDI-MS (Figure 1b in the Manuscript) shows the successful conjugation of one molecule of Dap-GRGDSP with two molecules of Acr-PEG-NHS.

Fabrication of hydrogels. $100 \mu \mathrm{l}$ of a solution containing 20\% (w/v) of PEGDA macromers and $0.05 \%(\mathrm{w} / \mathrm{v})$ of $\mathrm{I} 2959$ in PBS was added to each well of a 6-well stainless steel mold. The plate was placed under a UV lamp $\left(365 \mathrm{~nm}, 2-3 \mathrm{~mW} / \mathrm{cm}^{2}\right)$. After $10 \mathrm{~min}$ of UV irradiation, a hydrogel disk (diameter $10 \mathrm{~mm}$ and thickness $1 \mathrm{~mm}$ ) was obtained by lifting the mold plate. The swelling ratio $(\mathrm{q})$ of hydrogels was determined gravimetrically. The resulting hydrogel was placed in a vial containing $4 \mathrm{ml}$ of PBS and incubated under gentle shaking $(100 \mathrm{rpm})$ at $37^{\circ}$ for 48 hours. Excess fluid was removed and the wet gel was weighed $\left(\mathrm{W}_{\text {wet }}\right)$. The gel was then freeze-dried for about 48 hours to get the dried weight $\left(\mathrm{W}_{\mathrm{dry}}\right)$. Then, the swelling ratio $(\mathrm{q})$ was calculated by using the equation: $\mathrm{q}=\mathrm{W}_{\text {wet }} / \mathrm{W}_{\text {dry }}$.

Cell culturing. Human pulmonary artery smooth muscle cells (SMCs, passage 7, Cambrex) were seeded and allow to grow to confluence in a $75-\mathrm{cm}^{2}$ tissue culture polystyrene flask containing SMC 
growth media (SmGM-II, Cambrex) at $37^{\circ} \mathrm{C}$ and $5 \% \mathrm{CO}_{2}$. Confluent cells were trypsinized and resuspended in $9 \mathrm{ml}$ of SmGM-II. PEG hydrogel solution containing 20\% (w/v) PEG macromers and $0.05 \%(\mathrm{w} / \mathrm{v}) \mathrm{I} 2959$ initiator in PBS, was steriled by filtering through a $0.2-\mu \mathrm{m}$ nylon syringe filter (Fischer Scientific), and pipetted into a sterilized 6-well stainless steel mold (diameter 10mm, height 1 $\mathrm{mm})$. The mold was exposed to a $365-\mathrm{nm}$ wavelength UV light $\left(2-3 \mathrm{~mW} / \mathrm{cm}^{2}\right)$ for 10 minutes to form hydrogel disks, which were then transferred into a 12-well tissue culture plate (Falcon). A solution, containing 50,000 of SMCs suspended in $2 \mathrm{ml}$ of SmGM-II, was added to each well. SmGM-II was replaced 2 hours after seeding. Cells were imaged using phase contrast microscopy with 100x magnification. 